\title{
Use Of Online Structured Oral Examinations For Postgraduate Specialist And Subspecialist Certification Examinations During The COVID-19 Pandemic In South Africa
}

\author{
Vanessa C. Burch ( $\nabla$ vanessa.burch@uct.ac.za ) \\ University of Cape Town \\ Jessica K. McGuire \\ University of Cape Town \\ Francis Mbouaffou \\ Colleges of Medicine of South Africa
}

Flavia Senkubuge

University of Pretoria

Mike Sathekge

University of Pretoria

Eric Buch

University of Pretoria

Johannes J. Fagan

University of Cape Town

\section{Research Article}

Keywords:

Posted Date: March 7th, 2022

DOI: https://doi.org/10.21203/rs.3.rs-1351525/v1

License: (c) (1) This work is licensed under a Creative Commons Attribution 4.0 International License. Read Full License 


\section{Abstract}

Introduction: The COVID-19 pandemic prevented many medical specialist programmes across the world from conducting certification examinations. However, the Colleges of Medicine of South Africa (CMSA), which is responsible for conducting all specialist and sub-specialist certification examinations in South Africa, continued conducting examinations despite the pandemic.

Objectives: The study was conducted to determine the success and challenges of conducting SOEs using Zoom, with a special focus on acceptability of this examination format to candidates.

Method: The CMSA hosted 2933 candidates undertaking examinations for 95 diploma, specialist and sub-specialist qualifications. Of these, 2176 candidates participated in examinations requiring SOEs which amounted to 7226 individual Zoom calls. A survey was conducted during the first cycle of examinations in 2020 to assess the acceptability of the examination format to candidates.

Results: Pass rates ranged from $82 \%$ to $96 \%$ for the respective qualifications across two examination cycles. The overall pass rate for these 2 examination cycles was higher than that of the previous 6 examination cycles for the CMSA. The majority of 542 candidates who completed the survey felt that it was a fair examination method that tested their clinical reasoning and insight appropriately. They also felt that the case scenarios were of adequate complexity, and that the material was well presented.

Conclusion: This ambitious undertaking has demonstrated that virtual, case-based SOEs are a feasible and acceptable examination option. It is anticipated that the positive outcome of this study, the significant time and cost savings to candidates, examiners and the organization will motivate member colleges to support the ongoing use of virtual SOEs as part of the composite examination package.

\section{Introduction}

The COVID-19 pandemic prevented many medical specialist programmes across the world from conducting annual or bi-annual certification examinations.(1) However, the Colleges of Medicine of South Africa (CMSA), which is responsible for conducting all specialist and sub-specialist certification examinations in South Africa, continued conducting examinations despite the pandemic. This decision was motivated by the need to continue to deliver new specialist practitioners to the workforce, maintain access to specialist training for candidates awaiting pre-arranged entry into programmes, and the knowledge that $25 \%$ of specialist trainees (residents) are non-South African medical graduates who are independently funded (self-funded or foreign government supported). They come to South Africa to gain access to advanced training to improve healthcare in their home countries. This is almost universally not only a great financial sacrifice; but a personal one, as their families usually do not accompany them. Continued access to training is imperative for these individuals.

Conducting certification examinations during a pandemic with stringent local lockdown conditions was only deemed possible if all in-person examinations could be re-engineered and offered as virtual remote 
examinations. Given the limited global experience with virtual or remote specialist certification examinations prior to the pandemic,(2) the CMSA had to embark on a process of rapid redesign of examinations and implementation (lead time of four weeks). The organisation was motivated to pursue this undertaking since the literature suggested that success was achievable if candidate authentication, prevention of cheating, cybersecurity and technical problems could be effectively managed.(2)

Prior to the pandemic the CMSA, in co-operation with South African universities, had been modernising the assessment processes of residents in line with international best practice.(3) The CMSA offers examinations for 95 postgraduate qualifications hosted by 28 colleges (the newly formed College of Sport and Exercise Medicine did not participate in this round of examinations) representing all specialist and subspecialist disciplines practised in South Africa.(4) Historically, residency and senior residency programmes have required completion of logbooks of procedures and portfolios of training activities prior to entering exit-level certification examinations. These composite examination processes included a written component (modified essay questions, short answer questions, or single-best-answer multiple choice questions). Successful candidates were invited to an in-person, patient-based clinical examination, often followed by an in-person, unstructured oral examination (viva voce).

Over the past 15 years, the CMSA has been working towards modernising examination processes in its member colleges, with a specific focus on composite assessment packages which include the following three main components:(5)

1. Workplace based assessment (WBA): this is aimed at longitudinal assessment of clinical and practical procedural skills in the workplace. It is being developed in collaboration with the universities but will primarily be a university responsibility.

2. Written examinations: essay questions have been replaced by short answer questions and single best answer (SBA) multiple choice questions (MCQs) to determine theoretical knowledge.

3. Structured oral examinations (SOE): with the increasing adoption of WBA to ensure clinical and procedural skills competence, summative, in-person, patient-based examinations are being replaced by SOEs, which consist of standardized scripted questions with predetermined responses and a marking memorandum.(6) The questions are based on case scenarios which may include video clips, radiological imaging, and other laboratory data. The focus of these case scenarios is to assess clinical reasoning, judgement, insight and decision-making as they relate to comprehensive patient care.

The Covid-19 pandemic necessitated rapid, large-scale implementation of SOEs using Zoom Video Communications $\odot$ (Zoom), because it was not possible for candidates, examiners or patients to travel to and congregate at geographically distant academic hospitals at medical schools hosting traditional, inperson examinations.

\section{Objectives of the STUDY}


The study was conducted to determine the success and challenges of conducting SOEs using Zoom during the COVID-19 pandemic, with a special focus on acceptability of this examination format to candidates.

\section{Method}

The study was conducted during the examinations held between June 2020 and June 2021. During this period the CMSA conducted two 20-week cycles of specialist and sub-specialist certification examinations. These examinations were conducted during a state of national disaster, which imposed strict lockdown restrictions, including limited movement of the public between 09:00 and 21:00, a complete curfew between 21:00 and 06:00, closure of all business and non-essential services, including schools and universities, and no air travel or inter-provincial road travel except by emergency permit issue. (7)

\section{Participants}

The survey, which formed the basis of this study, was administered to the first cohort of candidates during the initial wave of the COVID-19 pandemic between June and November 2020. Candidates undertaking a SOE as part of fellowship or certificate certification examinations were invited to participate in the survey. Diploma candidates were not included in the study because the use of SOE for these candidates had not been finalised by the time this study was submitted for ethics committee approval.

\section{Study survey}

Participating candidates were requested to complete an online electronic survey (Addendum A) administered immediately after the onsite SOE. The survey was designed as a Google form and consisted of 21 questions which were answered using a binary (yes/no) response or a 5-point Likert scale (completely disagree, disagree, neutral, agree, completely agree). Five free text response questions were also included. The survey explored candidates' perceptions of the appropriateness and acceptability of both the mode of delivery (videoconference using Zoom) and format (SOE) of the virtual oral examinations.

For this study to be conducted as part of the examination proceedings it was decided to withhold collection of any demographic information (age, gender and ethnicity). Participants were only required to disclose which certification examination they had undertaken.

\section{Format of structured oral examinations}

SOEs were designed around case scenarios and consisted of short case descriptions with additional visual material in the form of photographs, videos clips and radiographic and histopathological images, where appropriate. The case scenarios were prepared by national panels of examiners working collaboratively on Zoom calls. Examination material for each case scenario was collated and presented as a Microsoft PowerPoint presentation to candidates on Zoom calls using the screen share facility. 
During each Zoom call, candidates reviewed the case scenarios and answered standardised questions posed by examiners working alone or in pairs. Zoom calls were also attended by moderators and trainee examiners. Examiners marked candidate responses using standardised memoranda and made notes during Zoom calls which were transcribed to electronic marksheets created in JotForm and submitted to the convener at the conclusion of the examination process. Each Zoom call was individually booked and collated on a timetable (embedded hyperlinks) which was shared with the examiner panel a week before the examination was due to take place. Zoom calls varied in length, ranging from 15-60 minutes, depending on the number of case scenarios discussed in each examination session. Candidates were assigned multiple case scenarios, ranging from 4-12 per session, depending on the specialty.

\section{Conduct of structured oral examinations}

Candidates were required to attend a CMSA approved examination venue to participate in the written and remote oral examination proceedings. To deal with the lockdown travel restrictions examination venues were set up in eight South African sites and six other African cities for candidates who were unable to travel to South Africa due to the pandemic. For this new examination process all the venues had to be equipped with laptop computers, 24-inch high-resolution monitors and internet connections using wireless, microwave and fibre technology. Owing to scheduled intermittent power interruptions in South Africa (load shedding),(8) all examination venues were also equipped with alternative power supply arrangements, which included diesel powered generators (large examination venues hosting more than 50 candidates) and uninterrupted power supply (UPS) units for computers and internet devices (all venues). Similar arrangements were secured at the examination venues outside South Africa. Onsite information technology support was available during all examinations and candidates were invigilated by trained staff at all examination venues, including sites outside South Africa. National occupational health and safety COVID-19 protocols, as applicable in the respective countries, were enforced throughout.(9)

\section{Data analysis}

The online survey data were collated and exported into a Microsoft Excel® spreadsheet prior to analysis. For questions using a Likert scale response, answers were reported in three categories: agree (strongly agree and agree responses), neutral (neutral responses) and disagree (strongly disagree and disagree responses). Percentages were calculated and rounded to one decimal point.

\section{Ethics approval}

The study and protocol were approved by the University of Cape Town's Human Research Ethics Committee (HREC 280/2020), the CMSA, the South African Committee of Medical Deans (SACOMD) and the Health Professions Council of South Africa (HPCSA). All examination candidates gave signed informed consent prior to participation in the study.

\section{Results}


Table 1 shows that between June 2020 and June 2021, the CMSA hosted 2933 candidates undertaking examinations for 95 diploma, specialist and sub-specialist qualifications. Of these, 2176 candidates participated in examinations requiring SOEs which amounted to 7226 individual Zoom calls over this period. 
Table 1

Examination characteristics of candidates during the 2 cycles of examinations

\begin{tabular}{|c|c|c|c|c|c|c|}
\hline Qualification & Year & $\begin{array}{l}\text { Diploma } \\
\text { (non- } \\
\text { specialist) }\end{array}$ & $\begin{array}{l}\text { Fellowship } \\
\text { (specialist) }\end{array}$ & $\begin{array}{l}\text { Certificate } \\
\text { (subspecialist) }\end{array}$ & Subtotal & Total \\
\hline \multirow[t]{2}{*}{ Participating colleges } & 2020 & 11 & 28 & 9 & & \multirow[t]{2}{*}{28} \\
\hline & 2021 & 11 & 28 & 10 & & \\
\hline \multirow[t]{2}{*}{ Qualifications offered } & 2020 & 12 & 36 & 43 & 91 & \\
\hline & 2021 & 14 & 36 & 36 & 86 & \\
\hline \multirow{2}{*}{$\begin{array}{l}\text { Candidates registering } \\
\text { for written component }\end{array}$} & 2020 & 498 & 762 & 112 & 1372 & \multirow[t]{2}{*}{3178} \\
\hline & 2021 & 706 & 947 & 1806 & & \\
\hline \multirow{2}{*}{$\begin{array}{l}\text { Candidates withdrawing } \\
\text { from written component }\end{array}$} & 2020 & 82 & 42 & 15 & 139 & \multirow[t]{2}{*}{233} \\
\hline & 2021 & 67 & 24 & 3 & 94 & \\
\hline \multirow{2}{*}{$\begin{array}{l}\text { Candidates passing } \\
\text { written component }\end{array}$} & 2020 & 416 & 717 & 97 & 1230 & \multirow[t]{2}{*}{2933} \\
\hline & 2021 & 636 & 917 & 150 & 1703 & \\
\hline \multirow{2}{*}{$\begin{array}{l}\text { Candidates passing } \\
\text { written component }\end{array}$} & 2020 & 338 & 587 & 81 & 1006 & \multirow[t]{2}{*}{2458} \\
\hline & 2021 & 533 & 787 & 132 & 1452 & \\
\hline \multirow{2}{*}{$\begin{array}{l}\text { Candidates withdrawing } \\
\text { from SOE }\end{array}$} & 2020 & 35 & 24 & 0 & 59 & \multirow[t]{2}{*}{134} \\
\hline & 2021 & 41 & 30 & 4 & 75 & \\
\hline \multirow{2}{*}{$\begin{array}{l}\text { Candidates undertaking } \\
\text { examinations without } \\
\text { SOE }\end{array}$} & 2020 & 70 & 16 & 6 & 92 & \multirow[t]{2}{*}{254} \\
\hline & 2021 & 122 & 18 & 22 & 162 & \\
\hline \multirow{2}{*}{$\begin{array}{l}\text { Candidates passing } \\
\text { written \& doing SOE }\end{array}$} & 2020 & 268 & 547 & 75 & 890 & \multirow[t]{2}{*}{2176} \\
\hline & 2021 & 411 & 769 & 106 & 1286 & \\
\hline \multirow[t]{2}{*}{ Candidates graduating } & 2020 & 290 & 497 & 75 & 862 & \multirow[t]{2}{*}{2124} \\
\hline & 2021 & 472 & 662 & 128 & 1262 & \\
\hline \multirow{2}{*}{$\begin{array}{l}\text { Pass rate of SOE } \\
\text { component of } \\
\text { examination process }\end{array}$} & 2020 & $82 \%$ & $88 \%$ & $92 \%$ & & \\
\hline & 2021 & $85 \%$ & $83 \%$ & $96 \%$ & & \\
\hline \multirow[t]{2}{*}{ Overall pass rate } & 2020 & $70 \%$ & $69 \%$ & $77 \%$ & & \\
\hline & 2021 & $74 \%$ & $72 \%$ & $85 \%$ & & \\
\hline
\end{tabular}


As seen in Table 1, most candidates chose to undertake the modified CMSA examinations offered during the initial waves of the COVID-19 pandemic. An exceptionally small proportion of candidates withdrew from the CMSA examinations once the new format had been announced (233/3166, 7.4\%). Furthermore, an even smaller number chose not to undertake SOEs once they had passed the written component of the respective examinations $(134 / 2310,0.6 \%)$. Also seen in Table 1 , most candidates who undertook SOEs were ultimately successful in passing their examinations; pass rates ranged from $82-96 \%$ for the respective qualifications across two examination cycles. The overall pass rate for these 2 examination cycles was higher than that of the previous 6 examination cycles for the CMSA (Table 2), particularly for the fellowship (specialty) and certificate (sub-specialty) examinations.

Table 2

Candidates who wrote examinations and the overall pass rate (\%) of the previous 6 semesters

\begin{tabular}{|lllllll|}
\hline & 2019 & & 2018 & & 2017 & \\
\hline Diploma, n (\%) & $469(79,3)$ & $306(75,5)$ & $419(75,2)$ & $290(71,3)$ & $346(74,6)$ & $275(70,9)$ \\
\hline Fellowship, n (\%) & $641(56,2)$ & $644(59)$ & $568(56,2)$ & $576(54,7)$ & $625(60)$ & $568(57,9)$ \\
\hline Certificate, n (\%) & $81(67,9)$ & $63(68,3)$ & $69(73,9)$ & $62(71)$ & $69(76,8)$ & $64(76,6)$ \\
\hline Total, n & 6135 & & & & & \\
\hline
\end{tabular}

The survey was conducted during the first cycle of examinations (2020), during which time 1230 candidates participated in written examinations of which 890 candidates proceeded to participate in SOEs via Zoom (June to November 2020). Only candidates participating in specialist and subspecialist certification examinations were recruited to the study (622 candidates). The study was launched during the first week of the 20-week examination cycle and 594 of 622 eligible candidates (95.5\%) were invited to participate. Of these 594 potential participants, $542(91.2 \%)$ completed the survey.

Table 3 shows that candidates generally had positive opinions regarding the use of SOEs by Zoom. The majority of 542 candidates who completed the survey in the first cycle of examinations conducted in 2020 felt that it was a fair method of examination (69\%), and that it tested their clinical reasoning and insight appropriately (73\%). They felt that the case scenarios were of adequate complexity $(77 \%)$, that the material was well presented (68\%), and that the larger number cases better displayed their knowledge $(67 \%)$. There were split opinions as to whether real patients would have improved the quality of the examination ( $n=542$, agree: $32 \%$, disagree: $37 \%$, neutral: $31 \%)$.

More than two-thirds of candidates thought that the length of the SOEs was appropriate $(n=542$, appropriate $67 \%$, too long $22 \%$, too short $11 \%$ ) and that the duration of individual stations/case discussions was adequate $(n=542$, appropriate $69 \%$, too long $3 \%$, too short $28 \%)$. 
Table 3

Candidates' opinions of structured oral examinations by Zoom (Positive opinions shaded grey)

\begin{tabular}{|c|c|c|c|}
\hline \multirow[t]{2}{*}{ Variables } & \multicolumn{3}{|c|}{$\begin{array}{l}\text { \% Level of agreement } \\
(n=542)\end{array}$} \\
\hline & Agree & Neutral & Disagree \\
\hline $\begin{array}{l}\text { The exam adequately tested my clinical reasoning, judgement, } \\
\text { insight, and decision-making }\end{array}$ & 73 & 20 & 7 \\
\hline $\begin{array}{l}\text { The case scenarios (examination questions) were appropriate to } \\
\text { assess an entry-level specialist / subspecialist }\end{array}$ & 77 & 17 & 6 \\
\hline The examination material was clearly presented & 68 & 19 & 13 \\
\hline $\begin{array}{l}\text { The use of a larger number of case scenarios rather than the } \\
\text { historically smaller number of cases gave me a better chance to show } \\
\text { my capability }\end{array}$ & 67 & 26 & 7 \\
\hline $\begin{array}{l}\text { Having real patients would have improved the quality of the } \\
\text { examination }\end{array}$ & 32 & 31 & 37 \\
\hline In my opinion, this was a fair examination & 69 & 24 & 7 \\
\hline
\end{tabular}

The results in Table 4 show that the Zoom platform that was used to conduct the SOEs was considered favourable by most candidates, who not only found it acceptable (80\%), but also reported that it was a fair examination technique (73\%). Most candidates reported that the examination process was technically adequate: $80 \%$ stated that they could clearly see and hear examiners and $69 \%$ said that video and image quality was adequate. SOEs conducted on Zoom was associated with personal financial $(80 \%)$ and time $(79 \%)$ benefits because candidates were spared the trouble of travelling. More candidates (43\%) preferred that examiners were present virtually, while $29 \%$ had no preference. Most participants (62\%) indicated that CMSA examinations should be conducted in the same manner in the future. 
Table 4

Candidates' opinions about the use of Zoom for conducting structured oral examinations (Positive opinions shaded grey)

\begin{tabular}{|c|c|c|c|}
\hline \multirow[t]{2}{*}{ Variables } & \multicolumn{3}{|c|}{$\begin{array}{l}\text { \% Level of agreement } \\
(n=542)\end{array}$} \\
\hline & Agree & Neutral & Disagree \\
\hline $\begin{array}{l}\text { I found it acceptable to have examiners conduct the examination } \\
\text { using Zoom }\end{array}$ & 80 & 15 & 5 \\
\hline $\begin{array}{l}\text { It would have been preferable to have a local examiner present with } \\
\text { me to ask the questions }\end{array}$ & 28 & 29 & 43 \\
\hline I could see the examiners clearly on the computer screen & 80 & 11 & 9 \\
\hline I could hear the examiners clearly on the Zoom call & 80 & 13 & 5 \\
\hline $\begin{array}{l}\text { Images and videos used were of adequate definition/quality to be } \\
\text { considered a fair examination }\end{array}$ & 69 & 20 & 11 \\
\hline $\begin{array}{l}\text { The personal "cost saving" of having the exam locally using Zoom } \\
\text { was worth it }\end{array}$ & 80 & 13 & 7 \\
\hline $\begin{array}{l}\text { The "time saved" by being able to participate in the } \\
\text { exam locally was worth it }\end{array}$ & 79 & 12 & 9 \\
\hline $\begin{array}{l}\text { Conducting oral examinations using Zoom is a fair } \\
\text { examination technique }\end{array}$ & 73 & 20 & 7 \\
\hline $\begin{array}{l}\text { The CMSA should continue to run the exams using Zoom as opposed } \\
\text { to a face-to-face process }\end{array}$ & 62 & 23 & 15 \\
\hline
\end{tabular}

A total 173 technical difficulties were reported during 7226 Zoom calls ( $2.4 \%$ of all calls), of which 164 were specifically described. Of these $44 \%$ were connectivity-related: delayed or poor connection, disconnection, laptop battery failure; $31 \%$ were sound-related: sound delay, poor quality or interruption, low sound intensity from examiners sitting too far from the microphone; $25 \%$ were image-related: video and/or photograph image quality (too small or unclear).

\section{Qualitative Data}

Additional information obtained from open-ended questions at the end of the survey showed that $24 \%$ of candidates $(n=130)$ specifically expressed appreciation for the level of organization of the examination, the professional and friendly behaviour of staff, examiners and invigilators and excellent implementation of COVID-19 sanitation protocols. Sixty-five (12\%) candidates also commented that the exam was fair and unbiased. Twelve percent of candidates $(n=65)$ reiterated the observation that the examination was less stressful because they didn't have to travel, which represented a saving in terms of both time and 
money. Suggestions for improvement included improving image quality of pictures and videos possibly by using projectors or televisions instead of laptop screens.

\section{Discussion}

This paper reports on the use of SOEs by Zoom Video Communications@ (Zoom) for postgraduate specialist and sub-specialist certification examinations conducted by the Colleges of Medicine of South Africa (CMSA) during the first two waves of the COVID-19 pandemic in 2020 and 2021. This included 7226 SOEs hosted on the Zoom platform for 95 qualifications hosted by 28 member colleges in the organisation. This method of examination, which has previously only been described in the literature as an appropriate and acceptable in-person assessment tool $(6,10-12)$, formed part of a composite assessment package which also included online written examinations and limited in-person clinical examinations. Rapid transition to this method of assessment during the initial stages of the COVID-19 pandemic enabled graduation of 2124 candidates despite severe lockdown restrictions.(7) To our knowledge this is the largest study conducted in the COVID-19 pandemic and the only study which reports on the use of virtual SOEs as part of national certification examinations for multiple specialties and subspecialties.

The bold approach taken by the CMSA, which ensured that specialist and sub-specialist certification processes were not postponed greatly assisted in preventing disruption of training programmes as experienced elsewhere in the world. $(2,13,14)$ The decision to conduct the certification examinations despite the pandemic was taken only 4 weeks before the first examination was conducted. Despite the incredibly short lead time to the examination, the CMSA was able to set up the required processes with the existing component of staff working remotely from home.

Although neither the candidates nor the examiners had any previous experience of virtual SOEs conducted on Zoom there was an overwhelmingly positive response to the examination process and a high level of acceptability reported by the candidates. Most found SOEs to be a fair and valid examination process, were satisfied with the use of Zoom for hosting the examination proceedings and endorsed its use for future examinations. This favourable outcome was facilitated by detailed preparation of support staff hosting the examination proceedings, extensive use of Zoom for training examiners and candidates, rapid electronic information dissemination and Zoom-based question and answer sessions to address the fears and anxieties of key stakeholders regarding the conduct of virtual SOEs. A major part of the preparation process was the development of standardised operating procedures to deal with technical problems such as internet disconnection, loss of Zoom calls and poor sound quality.(2)

In the examinations conducted by the CMSA, SOEs were predominantly used to assess clinical diagnostic reasoning, knowledge of patient care and communication skills. Research is currently seeking how best to use this examination process to assess additional clinical aptitudes including collaboration skills, leadership skills, health advocacy and professionalism(6, 10-12). In our examination proceedings, most 
candidates felt that paper-based cases were sufficient for the examination (as opposed to standardised patients or patient actors). This provides additional useful data about the format of SOEs for future users.

There is evidence that online assessment is a valid tool for assessing knowledge, and that it holds benefit to both the examiner and the candidate $(13,15)$. The challenge lies in its implementation. Technical issues have been highlighted as the major obstacle to conducting virtual examinations.(1) We experienced surprisingly few technical problems during the first 20-week examination cycle that formed part of this study. This may largely be ascribed to thorough preparation, multiple modes of IT connectivity, back-up electricity supplies and onsite IT support. Anyone wishing to replicate this model of assessment should pay particular attention to good quality internet connectivity and adequate information technology support during the examination. In addition, candidates should have a large screen on which to view recordings and images (a laptop computer screen is too small), and the examiners should wear microphones to improve sound quality.

In line with current literature, personal time and cost savings were reported as an additional advantage to the examination format.(2) In addition, this examination was accessible to international candidates who were not able to travel due to pandemic restrictions. The national and international travel and accommodation cost savings of this format of examination could be continued outside of pandemic conditions and would represent a significant ongoing advantage of this type of examination in the future. Improved access to examinations for international medical graduates completing postgraduate examinations remotely, at greatly reduced cost, may possibly represent a significant contribution to overall improvement of health care services in developing countries.

The initial expense to transition to the online examination process was a major financial hurdle. This outlay was offset by the usual cost of transporting and accommodating the pool of examiners from all over South Africa, as was done in traditional in-person examinations prior to the pandemic. There is currently no literature on the cost-effectiveness of remote SOEs compared to traditional in-person oral examinations and a detailed analysis is underway.

A major proportion of the cost of SOEs was the for use of onsite invigilation rather than remote proctoring. While remote proctoring technology has gained momentum during the pandemic $(16,17)$ we elected to use a traditional approach with onsite examination invigilation. This was motivated by the need for all candidates to have equal access to robust internet connectivity (multiple access routes), a secure electricity supply during the examination proceedings, and our limited experience of remote proctoring technology. The use of in-person onsite invigilation effectively addressed the risks of cheating and cybersecurity breaches. Going forward, remote proctoring is an attractive option, but we could only consider its use at established examination centres with robust internet connectivity and back up electricity sources.

The most significant limitation of virtual SOEs is the limited capacity of such examinations to assess hands-on clinical skills. In our context this was overcome by offering geographically decentralised, in- 
person assessment of clinical competence for specific specialities who deemed this a non-negotiable part of the assessment process. This is in keeping with a recent study reporting on a virtual orthopaedic clinical examination where the authors concluded that virtual examinations cannot replace the traditional examination format because candidates must examine patients or patient-actors during an examination(14). This is one of the priority reasons for the push to embed workplace-based assessment (WBA) in postgraduate training programmes in South Africa, which would fully address this issue in the future and obviate the need to verify clinical competence during exit-level specialist certification examinations.(18)

While this study focused on postgraduate specialist and subspecialist examinations in only one country, it does provide a broad overview of candidates' perceptions of the use of virtual SOEs by Zoom as an option for conducting examinations. These examinations were conducted in extraordinary circumstances which may have led to greater acceptability amongst candidates. So it would be important to determine ongoing acceptability outside of pandemic conditions.

\section{Conclusion}

This ambitious undertaking by the Colleges of Medicine of South Africa has demonstrated that virtual, case-based structured oral examinations are a feasible and acceptable examination option. The COVID19 pandemic provided the impetus for this dramatic alteration to a very traditional in-person examination process. It is anticipated that the positive outcome of this study, the significant time and cost savings to candidates, examiners and the organization will motivate member colleges to support the ongoing use of virtual SOEs as part of the composite examination package. The aggressive drive to introduce WBA and the ongoing use of geographically decentralised, in-person clinical examinations for selected specialties should further support the ongoing use of this new examination process.

\section{Declarations}

Additional files

Raw data - supplementary document

Acknowledgments

The authors wish to thank the candidates for participating in the study, as well as, a special thank you to Bianca van der Westhuizen and Riefda Thomson for the time they spent assisting the authors' with data extraction.

Funding

No funding was obtained for this study.

Availability of data and materials 
Attached as a supplementary file

Authors' contributions

VB conceptualisation of the study, proposal design, study implementation, data analysis, and manuscript preparation and review

JM literature review, data analysis, and manuscript preparation and review

EB conceptualisation of the study, proposal design, study implementation, data analysis

FM study implementation, data capture, data analysis and manuscript review

MS proposal design and manuscript review

FS proposal design and manuscript review

JF conceptualisation of the study, proposal design and submission, and manuscript preparation and review

Ethics approval and consent to participate

The study and protocol were approved by the University of Cape Town's Human Research Ethics Committee (HREC 280/2020), the CMSA, the South African Committee of Medical Deans (SACOMD) and the Health Professions Council of South Africa (HPCSA). The research, which involved human participants, was performed in accordance with the Declaration of Helsinki. All examination candidates gave signed informed consent prior to participation in the study. All methods were carried out in accordance with relevant guidelines and regulations.

Consent for publication

Declared consent for publication is not applicable as no identifying images or information was used in the manuscript.

Competing interests

The authors declare that they have no competing interests.

\section{References}

1. General Medical Council. The state of medical education and practice in the UK 2021: Experiences and challenges in postgraduate medical education [Internet]. 2021. Available from: https://www.gmcuk.org/-/media/documents/somep-2021-full-report_pdf-88509460.pdf?

la =en\&hash=058EBC55D983925E454F144AB74DEE6495ED7C98 
2. Ellis R, Oeppen RS, Brennan PA. Virtual postgraduate exams and assessments: the challenges of online delivery and optimising performance. British Journal of Oral and Maxillofacial Surgery. 2021 Feb 1;59(2):233-7.

3. Heeneman S, Jong LH de, Dawson LJ, Wilkinson TJ, Ryan A, Tait GR, et al. Ottawa 2020 consensus statement for programmatic assessment -1 . Agreement on the principles. Medical Teacher. 2021;43(10):1149-1160.

4. The Colleges of Medicine of South Africa: Colleges [Internet]. [cited 2021 Oct 4]. Available from: https://www.cmsa.co.za/view_college_list.aspx

5. The Colleges of Medicine of South Africa: Documents: Transactions [Internet]. [cited 2021 Oct 4]. Available from: https://www.cmsa.co.za/view_document_list.aspx?Keyword=Transactions

6. Jefferies A, Simmons B, Ng E, Skidmore M. Assessment of multiple physician competencies in postgraduate training: Utility of the structured oral examination. Advances in Health Sciences Education. 2011 Dec;16(5):569-77.

7. Read South Africa's full level 4 lockdown restrictions here [Internet]. [cited 2021 Sep 30]. Available from: https://businesstech.co.za/news/government/392831/read-south-africas-full-level-4-lockdownrestrictions-here/

8. Eskom details plans for winter load shedding in South Africa [Internet]. [cited 2021 Sep 5]. Available from: https://businesstech.co.za/news/energy/404615/eskom-details-plans-for-winter-load-shedding-insouth-africa/

9. Safety in the workplace / South African Government [Internet]. [cited 2021 Sep 5]. Available from: https://www.gov.za/covid-19/companies-and-employees/safety-workplace

10. Thiessen N, Fischer MR, Huwendiek S. Assessment methods in medical specialist assessments in the DACH region-overview, critical examination and recommendations for further development. GMS Journal for Medical Education. 2019;36(6). Available from: https://www.aerztekammer.at/]

11. Warner DO, Lien CA, Wang T, Zhou Y, Isaak RS, Peterson-Layne C, et al. First-year results of the American Board of Anesthesiology's objective structured clinical examination for initial certification. Anesthesia and Analgesia. 2020;1412-8.

12. Warner DO, Isaak RS, Peterson-Layne C, Lien CA, Sun H, Menzies AO, et al. Development of an Objective Structured Clinical Examination as a Component of Assessment for Initial Board Certification in Anesthesiology. Anesthesia and analgesia. 2020 Jan 1;130(1):258-64.

13. Pettit M, Shukla S, Zhang J, Sunil Kumar KH, Khanduja V. Virtual exams: has COVID-19 provided the impetus to change assessment methods in medicine? Bone \& Joint Open. 2021;2(2), 111-118. https://doi.org/10.1302/2633-1462.22.bjo-2020-0142.r1 
14. lyengar KP, Jain VK, Vaishya R. Virtual postgraduate orthopaedic practical examination: a pilot model. Postgraduate Medical Journal. BMJ Publishing Group. 2021, Oct 1. Available from: https://doi.org/10.1136/postgradmedj-2020-138726

15. Shebrain S, Nava K, Munene G, Shattuck C, Collins J, Sawyer R. Virtual Surgery Oral Board Examinations in the Era of COVID-19 Pandemic. How I Do It! Journal of Surgical Education. 2021 May $1 ; 78(3): 740-5$.

16. Andreou V, Peters S, Eggermont J, Wens J, Schoenmakers B. Remote versus on-site proctored exam: comparing student results in a cross-sectional study. BMC Medical Education 2021 21:1 [Internet]. 2021 Dec 20 [cited 2021 Dec 29];21(1):1-9. Available from: https://bmcmededuc.biomedcentral.com/articles/10.1186/s12909-021-03068-x

17. Morin M, Alves C, Champlain A de. The Show Must Go On: Lessons Learned from using Remote Proctoring in a High-stakes Medical Licensing Exam Program in Response to Severe Disruption. Journal of Applied Testing Technology [Internet]. 2021 Nov 30 [cited 2021 Dec 29];0(0). Available from: http://jattjournal.com/index.php/atp/article/view/165790

18. Sathekge MM. Work-based assessment: A critical element of specialist medical training. South African Medical Journal. 2017;107(9):728.

19. ten Cate O. Competency-Based Postgraduate Medical Education: Past, Present and Future. GMS Journal for Medical Education. 2017;34(5).

20. Caretta-Weyer HA, Chan T, Bigham BL, Kinnear B, Huwendiek S, Schumacher DJ. If we could turn back time: Imagining time-variable, competency-based medical education in the context of COVID-19. Medical Teacher. 2021;43(7):774-779.

\section{Supplementary Files}

This is a list of supplementary files associated with this preprint. Click to download.

- ZoomExamData13DEC2020.xlsx 